\title{
Comparison Group and Individual Cognitive Behavioral Therapy in Treatment of Depression among Iranian Women around Menopause
}

\author{
Robab Khoshbooii \\ Department of Guidance and Counseling, Science and Research Branch \\ Islamic Azad University, Tehran, Iran \\ E-mail: R.khoshbooii@yahoo.com
}

Received: November 23, 2011

Accepted: January 10, 2012

Published: March 1, 2012

doi:10.5539/ijps.v4n1p174

URL: http://dx.doi.org/10.5539/ijps.v4n1p174

\begin{abstract}
The aim of this study was to examine the effects of cognitive behavioral therapy (CBT) on depression and compare the difference between two formats of group and individual CBT among Iranian women at mid-life. The Sample for this study comprised 64 women (aged between 41 and 55 years with a depression score between 21 and 56) in Tehran, capital city of Iran. The participants were randomly assigned into two experimental groups and one control group. The first experimental group was exposed to 16 sessions of group CBT and the second experimental group underwent eight sessions of individual CBT. The experimental and control groups were assessed for the level of depression before and after the interventions and four weeks follow up. Descriptive statistics, Repeated Measure ANOVA, and independent $t$-test were used to analyze the data. The results suggested the feasibility and clinical advantages of CBT on depression but showed no significant difference between group and individual intervention among Iranian women around menopause.
\end{abstract}

Keywords: Group and individual cognitive behavioral therapy, Depression

\section{Introduction}

Depression is one of the most complicated and widespread disorders with serious consequences for individuals and societies. It is estimated that depression is the second most common psychological disorder which affects about 100 million people worldwide annually (Clark \& Beck, 1999). The World Health Organization projected that by 2020, depression will be the second almost prevalent non-infectious disease, and secondary only to ischemic heart disease (Murray \& Lopez, 1996). It is also estimated that the prevalence of disability brought about by depression is on the rise. According to the World Health Organization (WHO), depression represented the fourth leading cause of disability worldwide in 1990, but by the year 2020 it is expected to become the second leading cause of disability worldwide (Murray \& Lopez, 1996).

Previous studies are clear on the greater rate of depression among women than men. It was reported that the lifetime risk for developing depression was $7 \%-21 \%$ for women and significantly less, from $2.6 \%-12.7 \%$ for men (Clark \& Beck, 1999). This high prevalence is regardless of nationality or socio-economic level of population (Weissman, Bland, Canino, Faravelli, Greenwald, \& Hwu, 1996; Clark \& Beck, 1999). It is supposed that women's depression is multifactor. Biologically, women experience more changes during their lifetime, thus they may develop premenstrual dysphoric disorder, postpartum depression, and depression during transition to menopause. Socially women face more pressures due to their responsibility for multiple tasking and at the same time face more discrimination than men. These conditions may lead women to become more vulnerable to developing depression during their lifetime.

Mid-life is a critical developmental period which is characterized by biological and psychosocial changes in women. This stage of women's life is faced with the empty nest syndrome, menopause, and mid-life crisis. These factors have the potential for the development of psychological problems among some women at this stage of life. The literature however acknowledges an increases risk in the frequency of depression among women during menopausal transition. Epidemiological and longitudinal studies have indicated higher levels of depressive symptoms in perimenopausal women compared to those not yet entering menopause (Bromberger \& Assmann 
2003; Cohen \& Soares, 2006). The results of a longitudinal study conducted on women who were between 36 and 46 years-old, and who had never experienced an episode of major depression showed a higher rate of major depression among women who entered the perimenopausal transition as compared to women who remained at premenopausal period (Cohen \& Soares, 2006). Freeman, Sammel, Lin, and Nelson (2006) reported that entering the perimenopausal stage of life is associated with significantly higher rates of depression compared to premenopausal stage. More importantly, women with a history of postpartum depression, premenstrual syndrome and a family history of depression are at higher risk of developing major depression during transition to menopause.

There are not accurate statistics available on the prevalence of depression among Iranian women around menopause. Studies have only reported different results based on the varied population and measurements. The first National Mental Health Survey in 1999 on people aged 15 and above, revealed that about a fifth of the population in the study $(25.9 \%$ of the women and $14.9 \%$ of the men) suffer from psychological disorders (Noorbala , Bagheri-Yazdi,Yasmi \& Mohamad ,2004). A similar result was found in a study in 2001 on those aged 18 years and over, which estimated that the rate of mood disorders was $23.4 \%$ in women and $10.9 \%$ in men (Mohammadi, Rahgozar, Bagheri-Yazdi, Naghavi, Pour-Etemad, Amin, et al., 2003). Moreover, studies have reported a wide regional difference in the prevalence of depression among women in different provinces of Iran. In this regard, the number of women who suffer from depression varies, from $52.9 \%$ in Charmahal-va-Bakhtyari to $15.5 \%$ in West Azarbaijan provinces (Noorbala et al., 2004). Although, the exact extent of prevalence of depression among Iranian women around menopause period is unknown, a few studies have reported rates of depression ranging from 27\%-39.2\% among women aged 40-60 (Shohani \& Rasoli, 2007; Baghianimoghadam \& Aminian, 2007; Dawlatian, 2007).

Depression is betrayed by depressed mood, markedly diminished interest or pleasure in activities, disturbed sleep, poor appetite, low energy, feeling of worthlessness, and excessive guilt, poor concentration, recurrent thoughts of death or suicidal ideation with or without a plan (DSM-IV, 1994). The depressive symptoms can lead women to health risk behaviors, increase the risk of suicide, involvement in more physical ill-health, experience more disability, and unproductive work-hours. Adequate treatment with long term effects and low rate of relapse can lead an individual to a more productive life which has positive consequence for societies as well. In this regard, a course in psychotherapy, which can be as effective as antidepressant with less risk of relapse and side effects, may be preferred by many women. To reach these goals, further investigation and more creative solutions need to be examined.

One of the most empirically validated approaches for the treatment of depression is Cognitive Behavioral Therapy or CBT (Beck, Rush, Shaw, \& Emory 1979). CBT has been used as a short-term and structured intervention for treatment of depression in many populations and among various ages. Despite the general belief in the effectiveness of CBT, relatively few studies have evaluated the effectiveness of CBT among Iranian women at mid-life. Thus, the first aim of this study was to examine the effectiveness of CBT on depressed women around menopause.

In addition, factors like lack of trained therapists, which makes patients wait for long periods before receiving psychotherapy intervention and high cost of individually delivered CBT sessions, might discourage many patients from seeking treatment. Although research on the use of psychotherapy is widely focused on group or individual therapy, there has been little work done to compare the efficacy of these two treatment formats. Thus, the second aim of this study was to compare the effectiveness of group and individual CBT among Iranian women with depression around the period of menopause.

\section{Materials and Method}

\subsection{Participants}

The participants were recruited from four selected health centers in Tehran, Iran, who were referred for depressive symptoms. The sample was composed of 72 depressed women between the ages of 40 and 55 years. Depression was defined according to BDI-II of more than 17, and structural interview was based on the DSM-IV definition of clinical depression. Exclusion criteria were: those currently under medication or psychotherapy, previous experience of CBT, severe suicidal thoughts or psychotic depression, and severe illness. Participants were randomly assigned to group CBT (G-CBT), individual CBT (I-CBT), and control group (C-G). The study used an experimental design and a quantitative method of data collection. The score of pre-test was considered as a baseline for the study outcome. A total of eight participants did not attend the final assessment, so they were excluded from the data analysis. 


\subsection{Measure}

All participants responded to an Iranian translation of BDI-II three times, including pre-test (T1), post-test (T2), and four weeks follow-up (T3) and one demographic questionnaire.

\subsubsection{BDI-II Questionnaire}

BDI-II is the most widely-used questionnaire to screen for depression, following improvement of depression or use as baseline measurement (Gloaguen, Cottraux, Cucherat, \& Blackburn, 1998). BDI-II was translated into Persian by Hojat and Shapurian (1986) and standardized on the Iranian population. Ghasemzadeh, Mojtabai, Khamghadiri, and Ebrahimkhani, (2005) reported high internal consistency (alpha $=0.87$ ) and an acceptable test-re-test reliability $r=0.74$ of questionnaire. BDI-II is a 21 -item instrument, which is designed to measure the severity of symptoms of depression as listed in the American Psychiatric Association's Diagnostic and Statistical Manual of Mental Disorders, Fourth Edition (DSM-IV, 1994). Each item is rated in intensity from 0-3, with 0 indicating no depressive symptomatology, to 3 , indicating a severe level of symptomatology. Total scores range from 0 to 63. The classification of depression scores involves index score of $\leq 9$, which is considered to be normal range; a score of 10 to 15 shows minimal depressive symptomatology, while a score of 16-31 points shows mild depression, and a score of 32-47 indicates moderate depression. There is indication of severe depression when the score is 48-63 (Beck, Steer, \& Garbin, 1988).

\subsubsection{Demographic Questionnaire}

Questions on demographic characteristics were asked about age, educational level, number of children, income, job, physical health and history of depression.

\subsection{Procedure}

Participants were required to confirm in writing their agreement to participate in this research activity during their admission interview. The researchers developed a group and individual CBT program for treating women with depression on the basis of Iranian culture and middle-aged women's needs. The sessions' protocol was constructed based on the model developed by Beck et al. (1979), and group Cognitive Behavioral Therapy by White and Freeman (2000) with some adaptation to address the middle-aged women's needs. In general terms, both group and individual format of CBT received the same instructions and schedule based on Beck et al. (1979). This treatment condition consisted of 16 twice weekly two-hour sessions for group CBT and eight weekly one-hour sessions for individual CBT based on Beck's 20-session plans for cognitive therapy (Beck et al., 1979). Group CBT sessions had more specific instructions for each session than individual CBT. Individual sessions were flexible according to specific needs of participants but the general format and structures of CBT were applied in all group and individual sessions. Therapy sessions covered the main components of CBT as follows:

\subsubsection{Psycho-education}

Participants were enlightened on the many aspects of depression, familiarized with the cognitive model of depression, and also taught social skills and problem-solving strategies related to their problems.

\subsubsection{Cognitive Interventions}

Such cognitive interventions covered the subjects of automatic negative thoughts, cognitive distortions, dysfunctional assumptions, testing thoughts and beliefs, and alternative thoughts. In this part, the subject of attitude, false belief, and assumptions related to age-changes were discussed.

\subsubsection{Behavioral Intervention}

This included modification of behavior, self-monitoring of daily behavior, monitoring mood and activities, rating mood in relation to activities, introducing the concepts of mastery and pleasure in activity, graded task, and relaxation.

\subsubsection{Assigning Homework}

Homework assigned to enhance the skills taught in the sessions. It was a main component of the treatment. Each session included reviewing homework, setting the agenda, summarizing the sessions, getting feedback, and assisting participants to apply CBT skills regarding their personal problems. In this part, unrealistic thoughts and beliefs relating to their personal life stressors were challenged and possible solutions for alleviating depression were identified.

\subsubsection{Relapse Prevention}

Participants were taught relapse prevention, the signs of it and how to face it. 


\subsection{Control Group}

The control group did not receive any intervention till the end of the study. Participants in the control group were offered group CBT upon completion of the study, including assessment procedures (pre-post-follow-up) as followed by the participants in the experimental groups.

\section{Results}

\subsection{Demographic Information}

The subjects' demographic characteristics and homogeneity of variance in the three groups were as follows: Table 1 shows frequency of demographic variables in three groups. According to Table 1 frequency and percentage of education, employment, number of children, and age of the three groups were almost the same. Also the analysis of variances (ANOVA) showed that there was no significant difference between the variables of age $F(2,61)=.925, p=.402$, income $\mathrm{F}(2,61)=.116, p=.891$ and depression $\mathrm{F}(2,61)=.257, p=.774$, in the three groups. Leven's test indicated that variables of age, income, and depression, had equal variances between the three groups before the experiment. Thus, three groups were matched in terms of demographic variables and were homogenous in terms of the dependent variable. In term of descriptive analysis the mean age of sample was 49.28, standard deviation was 4.48, the mean depression scores was 33.48, and standard deviation 8.82 .

\subsection{Descriptive Statistics}

Table 2 presents the mean and standard deviations of depression in three measurements among participants of three groups. These results show that depression scores change over the time in the three groups.

\subsection{Within Subject Comparison}

Results of repeated measure ANOVA within groups are presented in Table 3. There is a statistically significant difference in the depression scores between T1, T2 and T3 at G-CBT group, $F(1,21)=269.60, p<.05$ and Cohen's $f=2.34$, at I-CBT group $F(1,19)=422.85, p<.05$, and Cohen's $f=4.24$. It is clear that depression scores significantly decrease across the three different time points among participants of the experimental groups, but there is no significant change for the control group. The obtained effect size indicates that the mean difference between the three measurements is very large, based on Cohen's (1992) criteria. On the basis of theses results, it can be concluded that group and individual CBT are effective interventions for treatment of depression.

\subsection{Comparisons between Groups}

An independent-samples $t$-test was conducted to compare the mean depression scores at T3 measurement between two formats of CBT. As Table 4 shows, the mean difference between two group is .88 , in which there is no significant $t(1,40)=.441, \mathrm{p}=.661$. These results indicate that there is no significant difference in the effectiveness of CBT between the two formats of group and individual therapy with regard to improvement of depression scores.

\section{Discussion}

In this research, the effort was to evaluate the effectiveness of cognitive behavioral therapy on depression in two formats of individual and group. The results from Tables 2 and 3 on the mean scores of depression across the three time points indicate that participants of the experimental groups demonstrate statistically significant improvement on the depression scores as measured by BDI-II across the time. A visual inspection of group means shows that participants of the group and individual CBT demonstrate a large decrease in depression score of 21.32 and 20.55 respectively. Findings regarding CBT's large treatment effect size in both the formats demonstrate the practical significance of CBT or its therapeutic value as an intervention for treatment of depression among middle-aged women. With regard to the depression scores in the control group, it can be concluded that changes in the level of depression in the experimental groups are the direct effect of principals of cognitive behavioral therapy. These results are comparable to previous studies on different populations in Iran which reported a significant reduction in participants' depression scores as a result of group or individual CBT (Moeenizadeh, 2010; Abolghasemi, 2008; Nilforooshan et al., 2006; Forozande, 2002) in Iran.

Additionally, the current results regarding the effectiveness of group CBT are supported by findings from previous studies. Oei and Dingle (2008) reviewed 34 papers and found that group cognitive behavior therapy is one of the most effective treatments for depression with effect sizes for G-CBT over the control group ranging from small (0.1) to large (2.87), with the average effect size of 1.10, indicating that the average person in a group cognitive behavior therapy condition is over one standard deviation more improved than the average person in the control condition by the end of treatment. Also, the pre-post treatment effect sizes for G-CBT range from 0.30 to 3.72 with the average effect size of 1.30 . Also, Clinical and long lasting effectiveness of individual CBT 
was examined in studies by Serfaty, Haworth and Buszewicz (2009) among 204 depressed individuals aged 65 years and older; and Hyun, Jung, and Jeong (2008) on 15 women with high BDI scores and high risk for developing postpartum depression. Also, the result of current study is comparable with studies by Henderson (2004), Rossello, Guillermo, and Carmen, (2008), Fujisawa, Nakagawa, and Miyuki (2010) that reported effectiveness of individual CBT among different populations.

The result from Table 4 shows that there is no significant difference between the two formats of treatment in relation to reduction of depression scores. Data achieved for mean differences is .88 , which is not statistically significant ( $p>05$ ). It means that group CBT and individual CBT, delivered by one therapist and applying the same structure and techniques resulted in the same outcomes in the experimental groups. These findings are supported by previous studies. McRoberts, Burlingame and Hoag (1998) reviewed 23 studies which were delivered by individual or group format. Results of this meta-analysis indicated that although, there was a little difference between group and individual therapy, this difference was not significant and the two formats of group and individual therapy have similar outcomes. Also, equivalent improvements on depression with no significant difference were reported by other studies (Henderson, 2004; Robinson et al., 1990; Oei \& Dingle, 2008; Rossello et al., 2008). The explanation for achieving the same results for group and individual CBT for improving depression is related to the use of the same principals of CBT at group and individual formats.

\section{Conclusion}

From the experience and insights gained through the first hypothesis of this study, it can be concluded that cognitive behavioral therapy (CBT) should be the treatment of choice for Iranian women at mid-life. Cognitive behavioral therapy (CBT) is close to Iranian culture and therapists can educate their clients on the logics of techniques and assumptions underlying them more easily. Thus, the clients can accept them with no trouble. Health professionals and policy makers may notice the effectiveness of cognitive behavior therapy and its adaptation to Iranian culture in the treatment of depression among women.

The second conclusion of this study confirms that group format of CBT is as effective as individual CBT. As reported in a previous study, the effect of G-CBT for treatment of depression is comparable with medication and other forms of psychotherapy including individual CBT (Oei \& Dingle, 2008). This finding may have implications on investigating group CBT as a cost-effective alternative to individual CBT. Cost-effectiveness of group therapy encourages and increases the number of patients willing to seek treatment for depression.

\section{References}

Abolghasemi. (2008). The effect of cognitive behavioal therapy on depression among clinically depressed individuals. Mashad, Iran. Jahade daneshgahi, 12(4), 108-112.

Baghiani Moghadam, M. H., \& Aminian, A. H. (2007). Depression in Menopaused and Non-menopaused Women. Journal of Research Health Science, 7 (2), 36-41.

Beck, A. T., Rush, A. J., Shaw, B. F., \& Emory, G. (1979). Cognitive Therapy of Depression. New York: Guilford Press.

Beck, A., Steer, R., \& Garbin, M. (1988). Psychometric properties of the Beck Depression Inventory: Twenty-five years of evaluation. Clinical Psychology Review, 8, 77-100. http://dx.doi.org/10.1016/0272-7358(88)90050-5

Beck, J. S. (1995). Cognitive Therapy: Basics and Beyond. New York: Guilford Press.

Bromberger, J. T., \& Assmann S. F. (2003). Persistent mood symptoms in a multiethnic community cohort of pre- and perimenopausal women. American Journal of Epidemiology, 158(4), 347-356. http://dx.doi.org/10.1093/aje/kwg155

Clark, D. M., \& Beck A. T. (1999). Scientific foundations of cognitive theory of depression. New York. John Wiley \& sons.

Cho H. J, Kwon J. H., \& Lee J. J. (2008). Antenatal cognitive-behavioral therapy for prevention of postpartum depression: a pilot study.

Cohen, L. S., \& Soares, C. N. (2006). Risk for new onset of depression during the menopausal transition, the Harvard study of moods and cycles. Archive of General Psychiatry, 63, 385-390. http://dx.doi.org/10.1001/archpsyc.63.4.385

Cohen, J. (1992). Quantitative methods in psychology: A power primer. Psychological Bulletin, 112(1), 155-159. http://dx.doi.org/10.1037/0033-2909.112.1.155 
Dawlatian (2007). The prevalence of depression among women of Kerman Shah (Iran) at menopause period. Jahade daneshgahi, 6(4), 78-84.

Dobson \& Dozois. (2001). Handbook of Cognitive behavioral Therapies. (second Ed. Book. New York: Guilford Press.

Dobson, Keith S. (1989). A meta-analysis of the efficacy of cognitive therapy for depression. Journal of Consulting and Clinical Psychology, 57(3), 414-419. Database: Psyc ARTICLES. http://dx.doi.org/10.1037/0022-006X.57.3.414

Freeman, E. W., Sammel, M. D., Lin, H., \& Nelson, D. B. (2006). Associations of hormones and menopausal status with depressed mood in women with no history of depression. Archive of General Psychiatry, 63(4), 375-82. http://dx.doi.org/10.1001/archpsyc.63.4.375

Frouzandeh, N. (2002). The effect of cognitive behavioral therapy on depression among college students of Shahre-cord. Jahade daneshgahi.

Fujisawa, D., Nakagawa, A., \& Miyuki, T. (2010). Cognitive behavioral therapy for depression among adults in Japanese clinical settings: a single-group study. BMC. Research Notes, 3, 160. http://dx.doi.org/10.1186/1756-0500-3-160

Gloaguen, V., Cottraux, J., Cucherat, M., \& Blackburn, I. M. (1998). A meta-analysis of the effects of cognitive therapy in depressed patients. Journal of Affective Disorders, 49, 59-72. http://dx.doi.org/10.1016/S0165-0327(97)00199-7

Ghasemzadeh, H., Mojtabai, R., Khamghadiri, N., \& Ebrahimkhani, N. (2005). Psychometric properties of a Persian-language version of the Beck Depression Inventory (2nf ed) BDI-II-PERSIAN. Depression and Anxiety, 21 (4), 185-192. http://dx.doi.org/10.1002/da.20070

Henderson, S. (2004). Effectiveness of group versus individual psychotherapy depression in a community counseling setting using Mind over Mood cognitive Therapy Manual. Unpublished doctoral dissertation, Calgray university, Alberta Canada

Hojat, M., \& Schapurian, R. (1986). Psychometric properties of a Persian version of the short form of the Beck Depression Inventory for Iranian college students. Psychological Reports, 59, 331-338. http://dx.doi.org/10.2466/pr0.1986.59.1.331

McRoberts, C., Burlingame, G. M., \& Hoag, M. J. (1998). Comparative efficacy of individual and group psychotherapy: a meta-analytic perspective. Group Dynamics: Theory, Research, and Practice. APA PsycNET, 2(2), 101-117.

Mohammadi M. R., Davidian H., Noorbala A. A., Malekafzali H., Naghavi H. R., \& Pouretemad H. R., et al. (2003). An epidemiological study of psychiatric disorders in iran. Hakim, 6(1), 55-64.

Moeenizadeh, M. (2010). Well-being therapy (WBT) for depression. International Journal of Psychological Studies, 2(1).

Murray, C. L., \& Lopez, A. D., (1998). The Global Burden of Disease: A Comprehensive Assessment of Mortality and Disability from Disease, Injuries, and Risk Factors in 1990 and Projected to 2020. Boston: Harvard press.

Murray, C. L., \& Lopez, A. D. (1996). The Global Burden of Disease and Injury Series, Volume 1 (Eds.) A Comprehensive Assessment of Mortality and Disability from Diseases, Injuries, and Risk Factors In 1990 and Projected to 2020. Cambridge, MA: P Harvard School of Public Health on behalf of the World Health Organization and the World Bank, Harvard University Press.

Nilforooshan, P., Ahmadi, A., Abedi, M. R., \& Ahmadi, M. (2006). Studying the effect of cognitive-behavioral counseling based on interacting cognitive subsystems on depression of infertile couples, Jahadde daneshgahi.

Noorbala, A. A., Bagheri Yazdi S. A., Yasamy M. T., \& Mohammad, K. (2004). Mental health survey of the adult population in Iran. Br J Psychiatry, 184, 70-73. http://dx.doi.org/10.1192/bjp.184.1.70

Oei T. P., \& Dingle G. (2008). The effectiveness of group cognitive behavioural therapy for unipolar depressive disorders. Journal of Affective Disorders, 107(1-3), 5-21. http://dx.doi.org/10.1016/j.jad.2007.07.018

Serfaty Marc Antony Haworth Deborah, Buszewicz, Marta (2009). Clinical Effectiveness of Individual Cognitive Behavioral Therapy for Depressed Older People in Primary Care. Arch Gen Psychiatry, 66 (12), 1332-1340. http://dx.doi.org/10.1001/archgenpsychiatry.2009.165

Shohani, M., \& Rasoli, F. (2007). Physical and psychological problems among menopausal women who refer to 
Ilam clinic. Jahade Daneshgahi, 21(5-4), 57-65.

Robinson, L. A., Berman, J. S., \& Neimeyer, R. A. (1990). Psychotherapy for the treatment of depression: a comprehensive review of controlled outcome research. Psychological Bulletin, 108, 30-49. http://dx.doi.org/10.1037/0033-2909.108.1.30

Rossello, J., Guillermo, B. L., \& Carmen, R. (2008). Individual and group CBT and IPT for Puerto Rican adolescents with depressive symptoms. Cultural Diversity and Ethnic Minority Psychology, 14(3), 234-245. http://dx.doi.org/10.1037/1099-9809.14.3.234

Sanai, B., Alaghband, S., Falahati, S., \& Hooman, A. (2009). Family and marriage scales. Tehran, Iran: Beast Publishing.

Spitzer, R. I., Williams, J. B. W., \& Gibson, M. (1990). User's Guide for Structured Clinical Interview for DSM-III-R(SCID). Biometrics Research Department, NewYork State Psychiatric Institute, New York.

Weissman, M. M., Bland, R. C., Canino, G. J., Faravelli, C., Greenwald, S., \& Hwu, H. G. (1996). Cross-national epidemiology of major depression and bipolar disorder. JAMA, 276(4), 293-9. http://dx.doi.org/10.1001/jama.276.4.293

Weiten, J. (2004). Introduction to Psychology. Massachusetts: Allen and Bacon.

White, J. R., \& Freeman, A. S. (2000). Cognitive-behavioral group therapy for specific problems and populations. Washington, DC: American Psychological Association. http://dx.doi.org/10.1037/10352-000

Table 1. Frequency of demographic variables in three groups $(\mathrm{N}=64)$

\begin{tabular}{|c|c|c|c|c|c|c|}
\hline \multirow{2}{*}{$\begin{array}{l}\text { Groups } \\
\text { Variables }\end{array}$} & \multicolumn{2}{|l|}{ C-G } & \multicolumn{2}{|l|}{ G-CBT } & \multicolumn{2}{|l|}{ I-CBT } \\
\hline & Frequency & Percent & Frequency & Percent & Frequency & Percent \\
\hline \multicolumn{7}{|l|}{ Education } \\
\hline$>$ diploma & 8 & 30 & 7 & 25 & 7 & 30 \\
\hline Diploma & 12 & 60 & 12 & 60 & 11 & 60 \\
\hline Bachelor & 2 & 10 & 3 & 15 & 2 & 10 \\
\hline \multicolumn{7}{|c|}{ Employment } \\
\hline Housewife & 22 & 25 & 22 & 25 & 22 & 25 \\
\hline Employee & 35 & 32.7 & 35 & 32.7 & 35 & 32.7 \\
\hline Retired & 7 & 22.2 & 7 & 22.2 & 7 & 22.2 \\
\hline \multicolumn{7}{|l|}{ Children } \\
\hline $0-2$ & 7 & 30.7 & 7 & 25 & 7 & 30 \\
\hline $3-6$ & 15 & 69.3 & 12 & 60 & 11 & 60 \\
\hline $6>$ & 0 & 0 & 3 & 15 & 2 & 10 \\
\hline \multicolumn{7}{|l|}{ Age } \\
\hline $40-45$ & 5 & 24 & 6 & 27 & 5 & 24 \\
\hline $46-50$ & 7 & 30 & 7 & 30 & 7 & 33 \\
\hline $51-55$ & 10 & 45 & 9 & 40 & 8 & 40 \\
\hline Total & 22 & 100 & 22 & 100 & 20 & 100 \\
\hline
\end{tabular}


Table 2. Descriptive of mean depression in three groups at three times

\begin{tabular}{|c|c|c|c|c|c|}
\hline Groups & $\mathbf{N}$ & $\begin{array}{l}\text { Mean \&SD } \\
\text { T1 }\end{array}$ & $\begin{array}{l}\text { Mean \& SD } \\
\text { T2 }\end{array}$ & $\begin{array}{l}\text { Mean \&SD } \\
\text { T3 }\end{array}$ & $\begin{array}{l}\text { Mean } \\
\text { difference } \\
\text { T1 \&T3 }\end{array}$ \\
\hline Group CBT(G-CBT) & 22 & $33.95(9.64)$ & $12.04(5.89)$ & $12.63(6.41)$ & 21.32 \\
\hline Individual CBT(I-CBT) & 20 & $32.30(8.73)$ & $10.85(6.17)$ & $11.75(6.59)$ & 20.55 \\
\hline Control group(C-G) & 22 & $34.09(8.34)$ & $32.77(6.92)$ & $33.77(7.17)$ & .32 \\
\hline Total & 64 & 33.48 & $18.79(11.6)$ & $19.62(12.27)$ & 13.86 \\
\hline
\end{tabular}

Table 3.Tests of within-subjects effects on depression over time in three groups (Repeated measure ANOVA)

\begin{tabular}{|l|clcccccc|}
\hline Groups & $\mathbf{N}$ & Source & T III SS* & df & MS** & F & Sig. & $\mathbf{f}^{* * *}$ \\
\hline G-CBT & 22 & Depression & 6855.36 & 1,21 & 5899.46 & 269.60 & .001 & 2.34 \\
I-CBT & 20 & Depression & 5888.10 & 1,19 & 4250.36 & 422.85 & .001 & 4.24 \\
CG & 22 & Depression & 20.81 & 1,21 & 15.68 & .54 & .517 & .02 \\
\hline
\end{tabular}

* Type III Sum of Squares $\quad * *$ Mean Square $\quad * * * \quad \mathrm{f}=$ effect size

Table 4. Independent $t$-test for comparing score of depression between two groups at T3

\begin{tabular}{|c|l|l|l|l|l|l|l|l|l|}
\hline \multicolumn{2}{|c|}{ Groups } & $\begin{array}{l}\text { Depression } \\
\text { Mean (SD) }\end{array}$ & \multicolumn{9}{|c|}{} & \multicolumn{2}{|c|}{$\begin{array}{l}\text { C5\% } \\
\text { Interval of of tence } \\
\text { Difference }\end{array}$} \\
\hline \multirow{2}{*}{ T3 } & G-CBT & $12.636(6.41)$ & t & df & Sig. p & $\begin{array}{l}\text { Mean } \\
\text { Difference }\end{array}$ & SD & Lower & Upper \\
\cline { 2 - 10 } & 1-CBT & $11.750(6.59)$ & .441 & 1,40 & .661 & .886 & 2.008 & -3.173 & 4.946 \\
\hline
\end{tabular}

